\title{
ON THE RELATIONSHIP BETWEEN ZERO ENTROPY AND QUASI-DISCRETE SPECTRUM FOR AFFINE TRANSFORMATIONS
}

PETER WALTERS

Introduction. If $T$ is a measure-preserving transformation of a Lebesgue space $(M, \beta, m)$ let $\pi(T)$ be the maximum partition such that $T_{x(T)}$ has zero entropy. In $\$ 2$ we prove a result, which was stated without proof in [12], concerning the behaviour of the partitions $\pi\left(T_{\zeta_{n}}\right)$ associated with an increasing sequence of invariant measurable partitions $\left\{\zeta_{n}\right\}$.

If $T$ is an ergodic affine transformation of a compact connected metric abelian group $G$ let $\eta(T)$ be the maximum partition such that $T_{\eta(T)}$ has quasi-discrete spectrum. In $\S 3$ we prove, using the result of $\S 2$ and a method introduced by Rohlin in [8], that $\eta(T)=\pi(T)$. This result was first obtained by Parry [6] who also proved that the maximum partition $\gamma(T)$ such that $T_{\gamma(T)}$ has the distal property is also $\pi(T)$.

My thanks are due to Dr. W. Parry for his guidance during this work.

\section{Preliminaries.}

1.1. The partition $\pi(T)$. For the definitions, notations and theory of Lebesgue spaces and entropy cf. [9], [10], [11].

Let $Z$ be the set of all measurable partitions of the Lebesgue space $(M, ß, m)$ which have finite entropy and let $T$ be a (possibly many to one) measure-preserving transformation of $(M, B, m)$. The maximum partition $\pi(T)$ such that $h\left(T_{\pi(T)}\right)=0$ is given by $\pi(T)=\bigvee_{\xi \in \mathcal{Z}}\left(\bigwedge_{n=1}^{\infty} T^{-n} \xi^{-}\right)$where $\xi^{-}=\bigvee_{k=1}^{\infty} T^{-k} \xi[11]$.

We have $T^{-1} \pi(T)=\pi(T), \pi\left(T^{n}\right)=\pi(T) n>0$, and if $T$ is one to one $\pi\left(T^{-1}\right)=\pi(T)$. If $\xi \in \mathcal{Z}$ and $\xi \leqq \pi(T)$ then $\xi \leqq \xi^{-}$.

$T$ is said to have completely positive entropy if $\pi(T)=\nu$, the trivial partition. If $T$ and $T^{\prime}$ are measure-preserving transformations of the Lebesgue spaces $(M, B, m)$ and $\left(M^{\prime}, \mathbb{B}^{\prime}, m^{\prime}\right)$ respectively and if there exists a measure-preserving transformation $\phi$ of $(M, B, m)$ onto $\left(M^{\prime}, \mathbb{B}^{\prime}, m^{\prime}\right)$ such that $\phi T=T^{\prime} \phi$, then $T^{\prime}$ is said to be a factor transformation of $T$ and $h\left(T^{\prime}\right) \leqq h(T)$ [11].

1.2. Affine transformations and group partitions. Let $G$ be a compact connected metric abelian group equipped with completed Haar measure. $G$ is a Lebesgue space [10]. All group operations are written

Received by the editors July 16, 1966. 
additively. Since $G$ is connected its character group $\Gamma$ has no elements of finite order [7]. Endomorphisms of $G$ and their duals will be denoted by the same symbol.

An affine transformation of $G$ is a transformation of the form $T(\mathrm{~g})$ $=a+A(g), g \in G$, where $a \in G$ and $A$ is a continuous endomorphism of $G$ onto $G$. Affine transformations preserve Haar measure. $T(g)=a$ $+A(g), g \in G$, is ergodic if and only if the smallest closed subgroup containing $a$ and $(A-I) G$ is $G$ and the only finite orbits under $A$ in the character group are fixed elements [3]. Since $G$ is connected, if $T$ is ergodic $T$ is totally ergodic (i.e., $T^{n}$ is ergodic for all $n>0$ ).

If $B G=G$, where $B=A-I$, then $T(b+g)=b+A(g), g \in G$, where $B(b)=-a$ and $T$ has completely positive entropy. (This follows from the fact that $A$ has completely positive entropy [8].)

Let $H$ be a subgroup of $G$. (All subgroups are assumed closed.) The measurable partition of $G$ into cosets of $H$ will be denoted by $\zeta(H)$ [8]. Such a partition is called a group partition. If $H_{1} \subset H_{2}$ then $\zeta\left(H_{1}\right) \geqq \zeta\left(H_{2}\right), \zeta(G)=\nu$, and $\zeta(0)=\epsilon$, the partition of $G$ into points. If $H_{1} \subset H_{2} \subset \cdots$ then $\zeta\left(\bigvee H_{n}\right)=\Lambda \zeta\left(H_{n}\right)$ where $\bigvee H_{n}$ denotes the smallest closed subgroup containing all the $H_{n}$, and if $H_{1} \supset H_{2} \supset \ldots$ then $\zeta\left(\Lambda H_{n}\right)=\vee \zeta\left(H_{n}\right)$ where $\Lambda H_{n}$ is the intersection of the groups $H_{n}$.

The partition $\alpha(T)=\bigwedge_{n=0}^{\infty} T^{-n} \epsilon$ is the finest partition of $G$ which satisfies $T^{-1} \alpha(T)=\alpha(T)$. In particular $\pi(T) \leqq \alpha(T) . \alpha(T)$ is a group partition since

$$
\alpha(T)=\alpha(A)=\bigwedge_{n=0}^{\infty} A^{-n} \epsilon=\zeta\left(\bigvee_{n=1}^{\infty} \text { kernel } A^{n}\right) .
$$

1.3. Affine transformations with quasi-discrete spectrum. The definition of a totally ergodic measure-preserving transformation with quasi-discrete spectrum is given in [1] and the definition of a homeomorphism with quasi-discrete spectrum is in [2]. Let $T(g)=a+A(g)$ be an ergodic affine transformation of a compact connected metric abelian group $G$ with character group $\Gamma$. If $\Gamma_{n}^{\prime}=\left\{\gamma \in \Gamma \mid B^{n} \gamma=0\right\}$ where $B=A-I$, then the group of quasi-eigenfunctions of order $n$ is $K \times \Gamma_{n}^{\prime}$ where $K$ is the circle group. $T$ has quasi-discrete spectrum if and only if $\bigcup_{n=1}^{\infty} \Gamma_{n}^{\prime}=\Gamma[4]$.

The maximum partition $\eta(T)$ such that $T_{\eta(T)}$ has quasi-discrete spectrum is the partition of $G$ into cosets of $\operatorname{ann}\left(\bigcup_{n=1}^{\infty} \Gamma_{n}^{\prime}\right)$ where $\operatorname{ann}\left(\bigcup_{n=1}^{\infty} \Gamma_{n}^{\prime}\right)$ is the annihilator of $\bigcup_{n=1}^{\infty} \Gamma_{n}^{\prime}$ i.e. $\eta(T)=\zeta\left(\operatorname{ann}\left(\bigcup_{n=1}^{\infty} \Gamma_{n}^{\prime}\right)\right)$ [4].

Abramov [1] has proved that every totally ergodic measure-preserving transformation of a Lebesgue space with quasi-discrete 
spectrum has zero entropy. Therefore for $T(g)=a+A(g), g \in G$, we have $\eta(T) \leqq \pi(T)$.

2. A limit theorem for $\pi(T)$. If $\zeta$ is a measurable partition of the Lebesgue space $(M, B, m)$ let $H_{\zeta}$ denote the projection of $M$ onto $M / \zeta$, i.e. $H_{\zeta}$ maps a point of $M$ onto the element of $\zeta$ to which it belongs. If $\zeta$ is a measurable partition of $M$ such that $T^{-1} \zeta \leqq \zeta$ then let $\pi^{\prime}\left(T_{\zeta}\right)$ denote $H_{\zeta}^{-1} \pi\left(T_{\zeta}\right)$.

THEOREM 1. Let $T$ be a measure-preserving transformation (possibly many to one) of a Lebesgue space $(M, \Theta, m)$ and let $\left\{\zeta_{n}\right\}$ be a sequence of measurable partitions of $M$ such that $\zeta_{1} \leqq \zeta_{2} \leqq \cdots, \bigvee_{n} \zeta_{n}=\epsilon$, and $T^{-1} \zeta_{n} \leqq \zeta_{n}$ for all $n$. Then $\pi^{\prime}\left(T_{\zeta_{1}}\right) \leqq \pi^{\prime}\left(T_{\zeta_{2}}\right) \leqq \cdots$ and $\mathrm{V}_{n} \pi^{\prime}\left(T_{\zeta_{n}}\right)=\pi(T)$.

Proof. Since $\pi^{\prime}\left(T_{\zeta_{n}}\right)=\pi(T) \wedge \zeta_{n}$ we have $\pi^{\prime}\left(T_{\zeta_{1}}\right) \leqq \pi^{\prime}\left(T_{\zeta_{2}}\right) \leqq \cdots$. Let $\pi_{1}(T)=\mathrm{V}_{n} \pi^{\prime}\left(T_{\zeta_{n}}\right)$.

Since $\pi^{\prime}\left(T_{\zeta_{n}}\right) \leqq \pi(T)$ for all $n$, we have $\pi_{1}(T) \leqq \pi(T)$. It remains to show that $\pi_{1}(T) \geqq \pi(T)$. Let $\xi \in \mathcal{Z}, \xi \leqq \pi(T)$ and $\eta \in \mathcal{Z}$. Then since $\xi \bigvee \xi^{-}=\xi^{-}$we have

$$
\begin{aligned}
H\left(\eta / \eta^{-}\right) & \geqq H\left(\eta / \eta^{-} \vee \xi\right) \geqq H\left(\eta / \eta^{-} \vee \xi \vee \xi\right)=H\left(\eta / \eta^{-} \vee \xi^{-}\right) \\
& =H\left((\eta \vee \xi) /\left(\eta^{-} \vee \xi\right)\right)=h(T, \eta \bigvee \xi) \geqq h(T, \eta)=H\left(\eta / \eta^{-}\right),
\end{aligned}
$$

i.e.

$$
H\left(\eta / \eta^{-} \bigvee \xi\right)=H\left(\eta / \eta^{-}\right)
$$

But

$$
H\left(\xi / \eta^{-}\right)+H\left(\eta / \xi \vee \eta^{-}\right)=H\left(\xi \vee \eta / \eta^{-}\right)=H\left(\eta / \eta^{-}\right)+H\left(\xi / \eta \vee \eta^{-}\right)
$$

and therefore

$$
H\left(\xi / \eta^{-}\right)=H\left(\xi / \eta \vee \eta^{-}\right) .
$$

Consequently $H\left(\xi / T^{-k} \eta^{-}\right)=H\left(\xi / \eta \bigvee \eta^{-}\right)$for all $k>0$, and if $\eta_{\infty}^{-}$ $=\bigwedge_{k=1}^{\infty} T^{-k} \eta^{-}$we have [9]

$$
H\left(\xi / \eta_{\infty}^{-}\right)=\lim _{k \rightarrow \infty} H\left(\xi / T^{-k} \eta^{-}\right)=H\left(\xi / \eta \vee \eta^{-}\right)
$$

Let $\xi \in \mathcal{Z}, \xi \leqq \pi(T)$ be fixed. Given $\delta>0, \exists \eta \in \mathcal{Z}, \eta \leqq \zeta_{n}$ for some $n$, such that $H(\xi / \eta)<\delta$.

Since $\eta_{\infty}^{-}=\bigwedge_{k=1} T^{-k} \eta^{-} \leqq \pi^{\prime}\left(T_{\zeta_{n}}\right) \leqq \pi_{1}(T)$, we have $H\left(\xi / \pi_{1}(T)\right)$ $\leqq H\left(\xi / \eta_{\infty}^{-}\right)=H\left(\xi / \eta \vee \eta^{-}\right) \leqq H(\xi / \eta)<\delta$. Therefore $H\left(\xi / \pi_{1}(T)\right)=0$ and $\xi \leqq \pi_{1}(T)$. Since this is true for all $\xi \in Z, \xi \leqq \pi(T)$, we have $\pi(T) \leqq \pi_{1}(T)$.

CoROllaRy. If $T$ is a measure-preserving transformation of a Lebesgue space $(M, \Theta, m)$ and if $\left\{\zeta_{n}\right\}$ is a sequence of measurable parti- 
tions of $M$ such that $\zeta_{1} \leqq \zeta_{2} \leqq \cdots, \vee_{n} \zeta_{n}=\zeta$, and $T^{-1} \zeta_{n} \leqq \zeta_{n}$ for all $n$, then $\mathrm{V}_{n} \pi^{\prime}\left(T_{\zeta_{n}}\right)=\pi^{\prime}\left(T_{\zeta}\right)$.

Proof. Apply Theorem 1 to the transformation $T_{\zeta}$ on $M / \zeta$.

\section{Zero entropy and quasi-discrete spectrum for affine transforma- tions.}

THEOREM 2. Let $G$ be a compact connected metric abelian group and let $T(g)=a+A(g), g \in G$, be an ergodic affine transformation of $G$. The maximum partition $\pi(T)$ such that $T_{\pi(T)}$ has zero entropy and the maximum partition $\eta(T)$ such that $T_{\eta(T)}$ has quasi-discrete spectrum coincide.

Proof. We first show that $\pi(T)=\eta(T)$ if, and only if, $\pi\left(T_{\alpha(T)}\right)$ $=\eta\left(T_{\alpha(T)}\right)$. From $\S 1.2 \alpha(T)=\zeta(F)$ for some subgroup $F$ of $G$ satisfying $A F=F$. Therefore $T_{\alpha(T)}$ is the one to one ergodic affine transformation $T_{\alpha(T)}\left(g^{\prime}\right)=a^{\prime}+A^{\prime}\left(g^{\prime}\right), g^{\prime} \in G / F$, where $A^{\prime}$ is the automorphism induced by $A$ in $G / F$ and $a^{\prime}$ is the coset of $F$ containing $a$. If $H_{\alpha(T)}$ is the projection map of $G$ on to $G / F$ then $H_{\alpha(T)}^{-1} \pi\left(T_{\alpha(T)}\right)=\alpha(T) \wedge \pi(T)$ and $H_{\alpha(T)}^{-1} \eta\left(T_{\alpha(T)}\right)=\alpha(T) \wedge \eta(T)$. Since $\eta(T) \leqq \pi(T) \leqq \alpha(T)$ we conclude that $\pi(T)=\eta(T)$ if and only if $\pi\left(T_{\alpha(T)}\right)=\eta\left(T_{\alpha(T)}\right)$.

We divide the proof of the theorem in to three parts.

(i) Suppose that $G$ is a finite-dimensional torus. We can suppose, without loss of generality, that $T$ and $A$ are one to one. This follows because $T_{\alpha(T)}$ is a one to one ergodic affine transformation of the finite-dimensional torus $G / F$ and $\pi(T)=\eta(T)$ if and only if $\pi\left(T_{\alpha(T)}\right)$ $=\eta\left(T_{\alpha(T)}\right)$.

The set of periodic points of an automorphism of a finite-dimensional torus is dense i.e. if $A_{n}=\left\{g \in G \mid A^{n} g=g\right\}, n>0$, then $\cup_{n=1}^{\infty} A_{n}$ is dense in $G$ [8]. If $\phi(g)=g_{1}+g$ where $g_{1} \in A_{n}$, then $\phi T^{n}=T^{n} \phi$ and hence $\phi^{-1} \pi\left(T^{n}\right)=\pi\left(T^{n}\right)$. Since $\pi\left(T^{n}\right)=\pi(T), n>0, \pi(T)$ is completely invariant under a dense set of translations and is therefore a group partition $\pi(T)=\zeta(N)$ [8].

$G_{1}=G / N$ is a finite-dimensional torus with character group $\Gamma_{1}=\operatorname{ann}(N)$, and $T_{\pi(T)}$ is the ergodic affine transformation $T_{1}(g)$ $=a_{1}+A_{1}(g), g \in G_{1}$, where $A_{1}$ is the automorphism of $G_{1}$ induced by $A$ and $a_{1}$ is the coset of $N$ containing $a$.

$\left\{B_{1}^{m} G_{1}, m \geqq 0\right\}$, where $B_{1}=A_{1}-I$, is a decreasing sequence of subgroups of $G_{1}$ and therefore $\left\{\operatorname{ann}\left(B_{1}^{m} G_{1}\right), m \geqq 0\right\}$ is an increasing sequence of subgroups of $\Gamma_{1}$. Since $\Gamma_{1}$ is a finitely generated free abelian group ann $\left(B_{1}^{n} G_{1}\right)=\operatorname{ann}\left(B_{1}^{n+1} G_{1}\right)$ for some $n$ and $B_{1}^{n} G_{1}=B_{1}^{n+1} G_{1}$.

Define an affine transformation $S$ on the compact connected metric abelian group $B_{1}^{n} G_{1}$ by $S\left(B_{1}^{n} g\right)=B_{1}^{n} a+A_{1}\left(B_{1}^{n} g\right), g \in G_{1}$. Since $A_{1}$ and $B_{1}$ commute we have $S B_{1}^{n}=B_{1}^{n} T_{1}$. Since $B_{1}\left(B_{1}^{n} G_{1}\right)=B_{1}^{n} G_{1}, S$ has com- 
pletely positive entropy (see $\$ 1.2$ ). But $h\left(T_{1}\right)=h\left(T_{\pi(T)}\right)=0$ and therefore $S$, being a factor transformation of $T_{1}$, has zero entropy (see \$1.1).

Therefore $B_{1}^{n} G_{1}=\{0\}$, and

$$
\Gamma_{n}^{\prime}=\operatorname{ann}\left(B_{1}^{n} G_{1}\right)=\left\{\gamma \in \Gamma_{1} \mid \gamma\left(B_{1}^{n} g\right)=0, \text { all } g \in G_{1}\right\}=\Gamma_{1},
$$

i.e. every element of $\Gamma_{1}$ is a quasi-eigenfunction of order $n$ (see $\S 1.3$ ), and therefore $T_{1}$ has quasi-discrete spectrum. Therefore $\eta(T) \geqq \pi(T)$ and hence $\eta(T)=\pi(T)$.

(ii) Suppose now that $\Gamma$, the character group of $G$, is finitely generated with respect to the endomorphism $A$, i.e. there exists a finite number of elements $\gamma_{1}, \cdots, \gamma_{r}$ of $\Gamma$ such that every element of $\Gamma$ is of the form $p_{1}(A) \gamma_{1}+\cdots+p_{r}(A) \gamma_{r}$ where $p_{i} \in \mathbb{Z}(A)$, the ring of polynomials in $A$ with integer coefficients.

We can again suppose, without loss of generality, that $T$ and $A$ are one to one. This follows since $\Gamma$ is a finitely generated module over the Noetherian ring $\mathrm{Z}(A)$ and is therefore a Noetherian module [13]. Since $\alpha(T)=\zeta(F)$, ann $(F)$ is a submodule of $\Gamma$ and hence is finitely generated over $\mathcal{Z}(A)$, i.e. there exists $\beta_{1}, \cdots, \beta_{s} \in \Gamma$ such that

$$
\text { ann }(F)=\left\{q_{1}(A) \beta_{1}+\cdots+q_{s}(A) \beta_{s} \mid q_{i} \in \mathbb{Z}(A), i=1, \cdots, s\right\} .
$$

Since $A(\operatorname{ann}(F))=\operatorname{ann}(F)$, ann $(F)$, the character group of $G / F$, is finitely generated with respect to $A_{\alpha(T)}$. Since $A_{\alpha(T)}$ and $T_{\alpha(T)}$ are one to one and $\pi(T)=\eta(T)$ if and only if $\pi\left(T_{\alpha(T)}\right)=\eta\left(T_{\alpha(T)}\right)$, the assertion follows.

Let

$$
\begin{aligned}
Y_{m}=\left\{p_{1}(A) \gamma_{1}+\cdots+p_{r}(A) \gamma_{r} \mid\right. & p_{i} \in \mathbb{Z}(A) \\
& \text { and degree of } \left.p_{i} \leqq m, i=1, \cdots, r\right\}
\end{aligned}
$$

$Y_{m}$ is a subgroup of $\Gamma$ and $\bigcup_{m=0}^{\infty} Y_{m}=\Gamma$. Choose $m$ such that $A^{-1} \gamma_{i} \in Y_{m}, i=1, \cdots, r$. Then $A^{-1} Y_{m} \subset Y_{m}$ and if $H=\operatorname{ann}\left(Y_{m}\right)$, $A^{-1} H \subset H$. Since $A^{n} Y_{m}, n \geqq 0$, are finitely generated with no elements of finite order, $G / A^{-n} H, n \geqq 0$, are finite-dimensional tori. We have $\bigcup_{n=0}^{\infty} A^{n} Y_{m}=\Gamma, \quad \bigcap_{n=0}^{\infty} A^{-n} H=\{0\} \quad$ and $\quad \zeta(H) \leqq \zeta\left(A^{-1} H\right) \leqq \cdots$, $\bigvee_{n=0}^{\infty} \zeta\left(A^{-n} H\right)=\epsilon$. Since $\left(T^{-1}\right)_{\zeta\left(A^{-n} H\right)}$ is an ergodic affine transformation of $G / A^{-n} H$ we have $\pi(T)=\pi\left(T^{-1}\right)=\bigvee_{n=0}^{\infty} \pi^{\prime}\left(\left(T^{-1}\right)_{\zeta\left(A^{-n} H\right)}\right)$ by Theorem 1, and if $\Gamma_{k}^{\prime}=\left\{\gamma \in \Gamma \mid B^{k} \gamma=0\right\}$ where $B=A-I$, then by part (i)

$$
\pi^{\prime}\left(\left(T^{-1}\right)_{\zeta\left(A^{-n} H\right)}\right)=\zeta\left(\operatorname{ann}\left(\bigcup_{k=1}^{\infty} \Gamma_{k}^{\prime} \cap A^{n} Y_{m}\right)\right) \quad(\text { see } \S 1.3) .
$$


Therefore

$$
\begin{aligned}
\pi(T) & =\bigvee_{n=0}^{\infty} \zeta\left(\operatorname{ann}\left(\bigcup_{k=1}^{\infty} \Gamma_{k}^{\prime} \cap A^{n} Y_{m}\right)\right) \\
& =\zeta\left(\operatorname{ann}\left(\bigcup_{k=1}^{\infty} \Gamma_{k}^{\prime}\right)\right)=\eta(T) .
\end{aligned}
$$

(iii) Suppose now that $G$ is a general compact connected metric abelian group. $\Gamma$ is countable, $\Gamma=\left\{\gamma_{1}, \gamma_{2}, \ldots\right\}$. Let $Y_{n}$ be the smallest subgroup of $\Gamma$ containing $\gamma_{1}, \cdots, \gamma_{n}$ and invariant relative to $A$ (i.e. $\left.A Y_{n} \subset Y_{n}\right)$. If $H_{n}=\operatorname{ann}\left(Y_{n}\right), A H_{n} \subset H_{n}$ and $T^{-1} \zeta\left(H_{n}\right) \leqq \zeta\left(H_{n}\right)$. We have

$$
\begin{aligned}
& Y_{1} \subset Y_{2} \subset Y_{3} \subset \cdots, \quad \bigcup_{n=1}^{\infty} Y_{n}=\Gamma \\
& H_{1} \supset H_{2} \supset H_{3} \supset \cdots, \quad \bigcap_{n=1}^{\infty} H_{n}=\{0\},
\end{aligned}
$$

and

$$
\zeta\left(H_{1}\right) \leqq \zeta\left(H_{2}\right) \leqq \zeta\left(H_{3}\right) \leqq \cdots, \quad \bigvee_{n=1}^{\infty} \zeta\left(H_{n}\right)=\epsilon .
$$

Using the fact that $Y_{n}$ is finitely generated with respect to the endomorphism $A_{\zeta\left(H_{n}\right)}$ we have $\pi^{\prime}\left(T_{\zeta\left(H_{n}\right)}\right)=\zeta\left(\operatorname{ann}\left(\bigcup_{k=1}^{\infty} \Gamma_{k}^{\prime} \cap Y_{n}\right)\right)$ by part (ii) where $\Gamma_{k}^{\prime}=\left\{\gamma \in \Gamma \mid B^{k} \gamma=0\right\}, B=A-I$. (See $\S 1$.3.) Therefore by Theorem 1

$$
\begin{aligned}
\pi(T)=\mathrm{V}_{n} \pi^{\prime}\left(T_{\zeta\left(H_{n}\right)}\right) & =\bigvee_{n} \zeta\left(\operatorname{ann}\left(\bigcup_{k=1}^{\infty} \Gamma_{k}^{\prime} \cap Y_{n}\right)\right) \\
& =\zeta\left(\operatorname{ann}\left(\bigcup_{k=1}^{\infty} \Gamma_{k}^{\prime}\right)\right)=\eta(T) .
\end{aligned}
$$

This completes the proof of the theorem.

REMARK. To prove part (ii) we could have used a result of Laxton and Parry [5], which states that an automorphism $A$ of a compact connected metric abelian group has a dense set of periodic points if the character group is finitely generated with respect to $A$.

\section{REFERENCES}

1. L. M. Abramov, Metric automorphisms with quasi-discrete spectrum, Izv. Akad. Nauk SSSR Ser. Mat. 26 (1962)=Amer. Math. Soc. Transl. (2) 39 (1964), 37-56.

2. F. J. Hahn and W. Parry, Minimal dynamical systems with quasi-discrete spectrum, J. London Math. Soc. 40 (1965), 309-323. 
3. A. H. M. Hoare and W. Parry, Semi groups of affine transformations, Oxford Quart. J. Math. 17 (1966), 106-111.

4. - Affine transformations with quasi-discrete spectrum, J. London Math. Soc. 41 (1966), 88-96.

5. P. R. Laxton and W. Parry, On the periodic points of certain automorphisms and a system of polynomial identities (to appear).

6. W. Parry, On the coincidence of three invariant $\sigma$-algebras associated with an affine transformation, Proc. Amer. Math. Soc. 17 (1966), 1297-1302.

7. L. Pontrjagin, Topological groups, Princeton Univ. Press, Princeton, N. J., 1946.

8. V. A. Rohlin, Metric properties of endomorphisms of compact abelian groups, Izv. Akad. Nauk SSSR Ser. Mat. 28 (1964). (Russian)

9. - Exact endomorphisms of a Lebesgue space, Izv. Akad. Nauk SSSR Ser. Mat. 25 (1961), 499-530=Amer. Math. Soc. Transl. (2) 39 (1964), 1-36.

10. - On the fundamental ideas of measure theory, Mat. Sb. 25 (1949), 107-150 = Amer. Math. Soc. Transl. (1) 10 (1962), 1-54.

11. - New progress in the theory of transformations with an invariant measure, Uspehi Mat. Nauk 15 (1960), 3-26= Russian Math. Surveys 15 (1960), 1-22.

12. S. A. YuzvinskiY, Metric properties of endomorphisms of compact groups, Izv. Akad. Nauk SSSR Ser. Mat. 29 (1965), 1295-1328.

13. B. L. van der Waerdon, Modern algebra, Vol. II, Ungar, New York, 1950.

University of Sussex, England 